\title{
The contour method for residual stress determination applied to an AA6082-T6 friction stir butt weld
}

\author{
V. Richter-Trummer ${ }^{1, a}$, P. M. G. P. Moreira ${ }^{2, b}$, J. Ribeiro ${ }^{2, c}$, P. M. S. T. de \\ Castro $^{1, d}$ \\ ${ }^{1}$ FEUP, Rua Dr. Roberto Frias, 4200-465 Porto, Portugal \\ ${ }^{2}$ INEGI, Campus da FEUP, Rua Dr. Roberto Frias, 4200-465 Porto, Portugal \\ ${ }^{a}$ valentin@fe.up.pt, ${ }^{b}$ pmgpm@fe.up.pt, ${ }^{c}$ jribeiro@ipb.pt, ${ }^{d}$ ptcastro@fe.up.pt
}

Keywords: contour method, friction stir welding, incremental hole drilling technique, residual stress, surface mapping

\begin{abstract}
Residual stresses parallel to the welding direction on a cross-section of a $3 \mathrm{~mm}$ thick friction stir butt-welded aluminium alloy AA6082-T6 plate were determined using the contour method. This is a destructive relaxation based measurement technique capable of determining the full-field residual stresses perpendicular to a plane of interest.

A wire electro discharge machining cut was performed revealing the plane of interest. The residual stresses present before the straight cut lead to a deformed cutting plane. Then, a coordinate measuring machine was used to acquire the cutting plane shape of both plate halves after the cut. A data reduction scheme for noise and error elimination was used. The measured deformation was applied to a linear elastic finite elements model considering the real specimen geometry.

A full contour map of longitudinal residual stresses on a weld cross section was determined in this way, revealing detailed information on the residual stress distribution in the inside of a friction stir weld, especially in the nugget zone. A typical M-shape, usually described for the residual stress distribution in friction stir welds, was found. The maximum residual stresses are below the yield strength of the material in the shoulder region and, outside of the welding region, low tensile and compressive residual stresses are responsible for the necessary stress equilibrium on the plane of interest.

A comparison was made with the established incremental hole drilling technique on an equivalent plate for validation and good agreement of both techniques was obtained. The distribution, as well as the magnitude of the residual stresses measured by both techniques, is very similar, thus validating both the experimental and numerical procedures used for the contour method application, which is presented and discussed in the paper.
\end{abstract}

\section{Introduction}

The contour method is a recent fully destructive residual stress measurement technique, developed by Prime in 2001 [1]. With this technique it is possible to determine residual stress state on a surface inside a body in the direction perpendicular to the cutting plane.

This technique has been used in a great variety of situations, but information regarding thin plates is rare [2]. The distorted shape of welded plates and the low displacements measurable 
Table 1: FSW parameters

\begin{tabular}{ll}
\hline Parameter & Value \\
\hline Welding speed & $290 \mathrm{~mm} / \mathrm{min}$ \\
Rotation speed & $1500 \mathrm{rpm}$ \\
Plate thickness & $3.02 \mathrm{~mm}$ \\
Penetration & $2.97 \mathrm{~mm}$ \\
Shoulder & channeled, $\phi=15 \mathrm{~mm}$ \\
Pin & conical,$\phi=5 \mathrm{~mm}$ \\
\hline
\end{tabular}

after the cut are challenges that need to be overcome.

The incremental hole drilling technique (iHDT), ASTM standard E837 [3], was also applied to a similar plate for validation purposes. This well established technique is able to measure the depth variation at a point of the residual stresses in various directions. 10 holes were drilled in order to be able to map a part of the residual stress distribution of the plate.

When residual stresses are high enough to cause yielding during a cut, the elastic superposition principle used by this technique is not applicable anymore. Shin [4] notes that the plasticity induced error is significantly lower in the case of the contour method than in the case of the hole drilling technique when residual stresses near the yield strength are to be measured. Proper constraining of the plate during the cut reduce this error further. In the present work, all efforts were made to adequately clamp the plate during the cutting process, and considering the measured stress magnitude according to Shin an error well below $5 \%$ is expected [4].

The longitudinal through-the-thickness residual stress distribution on a friction stir welding (FSW) cross section has been determined for a $3 \mathrm{~mm}$ thick aluminium plate. Welding was performed under displacement control with the welding parameters shown in Table 1.

The material used was the aluminium alloy AA6082-T6, with a Young's modulus (E) of 65 GPa and a yield strength of $252 \mathrm{MPa}[5]$.

\section{Experimental setup}

This residual stress determination method is divided into four parts. First a wire electro discharge machine (wEDM) cut has to be performed revealing the plane of interest, being the determined stresses perpendicular to this plane. Secondly, a coordinate measuring machine is used for determination of the surface relaxation after the cut. The measured data is treated with a data reduction scheme for noise and error elimination. A finite element model is built considering the real geometry and the measured deformation is applied to the model so that stresses on the surface may be retrieved. 
wEDM cutting of the specimen The cut was done on a Sodick wEDM at Autoconceptus in Rio Tinto, Portugal. A $0.25 \mathrm{~mm}$ diameter wire was used for the cutting process, and the cutting speed was about $24 \mathrm{~mm} / \mathrm{min}$. The cut was made at once.

Plate half A has a length of $126 \mathrm{~mm}$ and plate half B measures $180 \mathrm{~mm}$. Figure 1 shows the cutting procedure and clamping arrangement. While it was tried to optimize the clamping arrangement, the cut was made with the only one half of the plate rigidly clamped due restrictions of the available equipment.

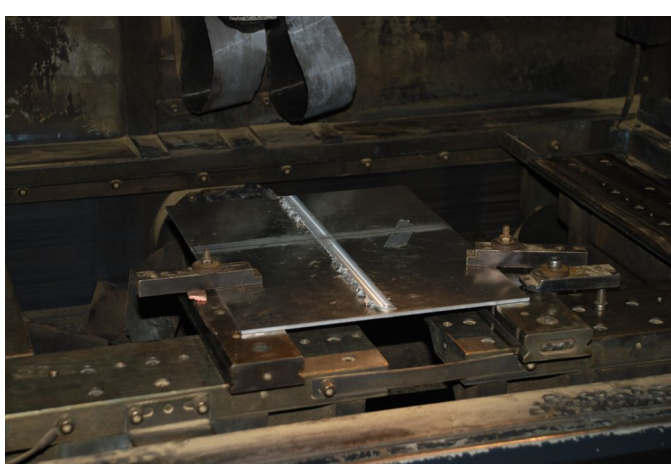

(a) Clamping arrangement

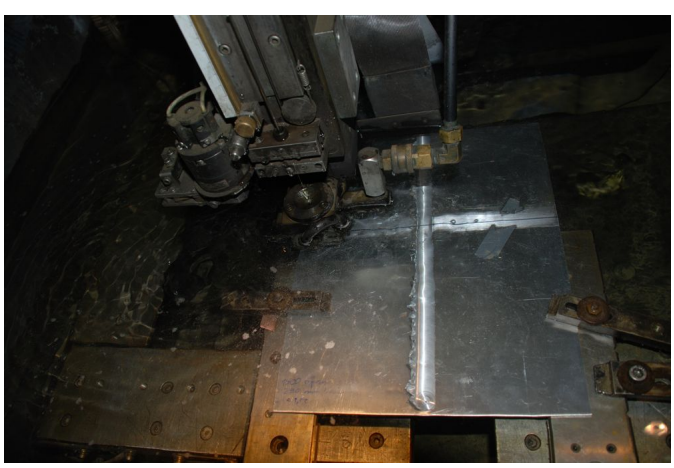

(b) Cutting procedure

Fig. 1: Experimental setup during the cutting process

Measurement of the cutting plane Measurements where performed at CATIM - Porto. Approximately 2000 points were measured on the surface of interest, and the shape of the plate was acquired for easier finite element modeling of the plate. The measurement of each plate half took approximately $5 \mathrm{~h}$.

Figure 2 shows the measurement procedure and clamping arrangement for one of the plate halves.

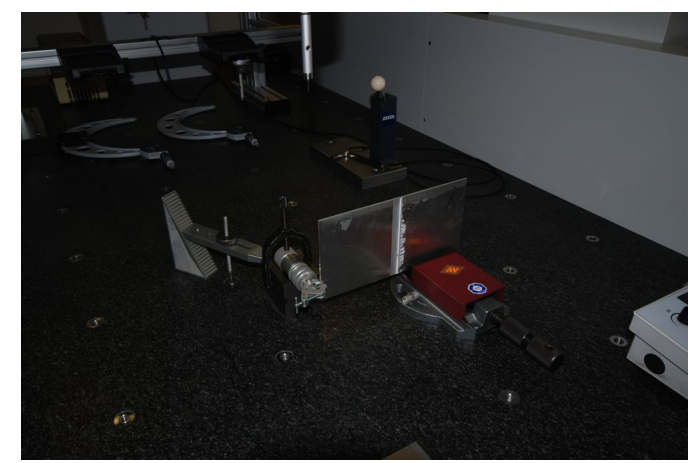

Fig. 2: Experimental setup during the CMM measurements 
More points have been acquired in the center of the plate than on the borders in the plate width direction, since a stronger variation is expected in the center, which leads to shorter measurement times.

Plate half B has a much lower surface deformation than plate half $\mathrm{A}$. Reasons may include the cutting path, which may differ slightly from a straight line. Plate half B is smaller, and has therefore less elastic energy stored due to a certain amount of residual stresses which is released during the cut. Ideally both plate halves would be securely clamped during the cutting process, but in the present case only plate half $\mathrm{A}$ was restrained during the cutting process. These factors reinforce the importance of averaging the surface deformation measurements carried out on both parts.

\section{Data conditioning}

Data conditioning is divided into three main steps. First the deformed surfaces have to be aligned with a horizontal plane, because there is no guarantee that this was the case during measurement. Afterwards, the average deformation value of each measured point has to be calculated in order to eliminate errors based on a non-straight cut. The result of both steps is shown in Figure 3, where both measured plate halves and their average deformation are represented.

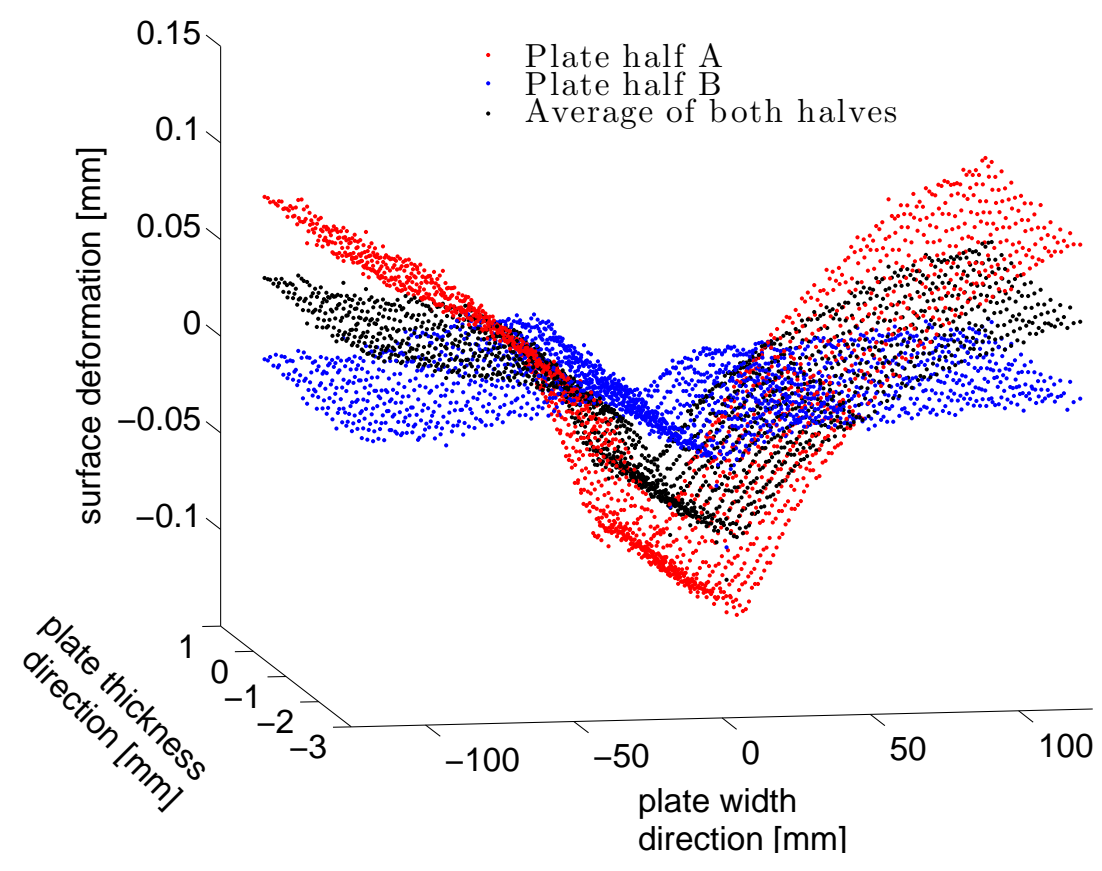

Fig. 3: Measured surface deformation after the first steps of the data conditioning process 
The average point cloud has to be smoothed to a surface which is continuous at least up to its first derivative for application in a finite element model. Therefore the SPAP2 function in Mathworks MatLab is used.

Quadratic splines have been chosen for the approximation, since they guarantee a continuous surface up to the first derivative which is needed for the finite element method (FEM). 23 knots where applied in $\mathrm{X}$ direction and 1 in $\mathrm{Y}$ direction. In $\mathrm{X}$ direction, a higher knot density was applied between $\pm 50 \mathrm{~mm}$ around the welding line than at the plate borders. It should be noted that a higher knot density tends to lead to higher noise in the stress results, since the calculation of stresses based on displacements is based on derivation, and therefore undulations in the displacement field will lead to stronger variations in the stress field. The knot number is therefore optimized, reducing it to the minimum possible number which still guarantees a good surface fit quality. A rectangular grid with 2000x20 points was overlaid on the measured points for use of the smoothing function. Figure 4 shows the smoothed surface based on the average displacement of both measured plate halves.

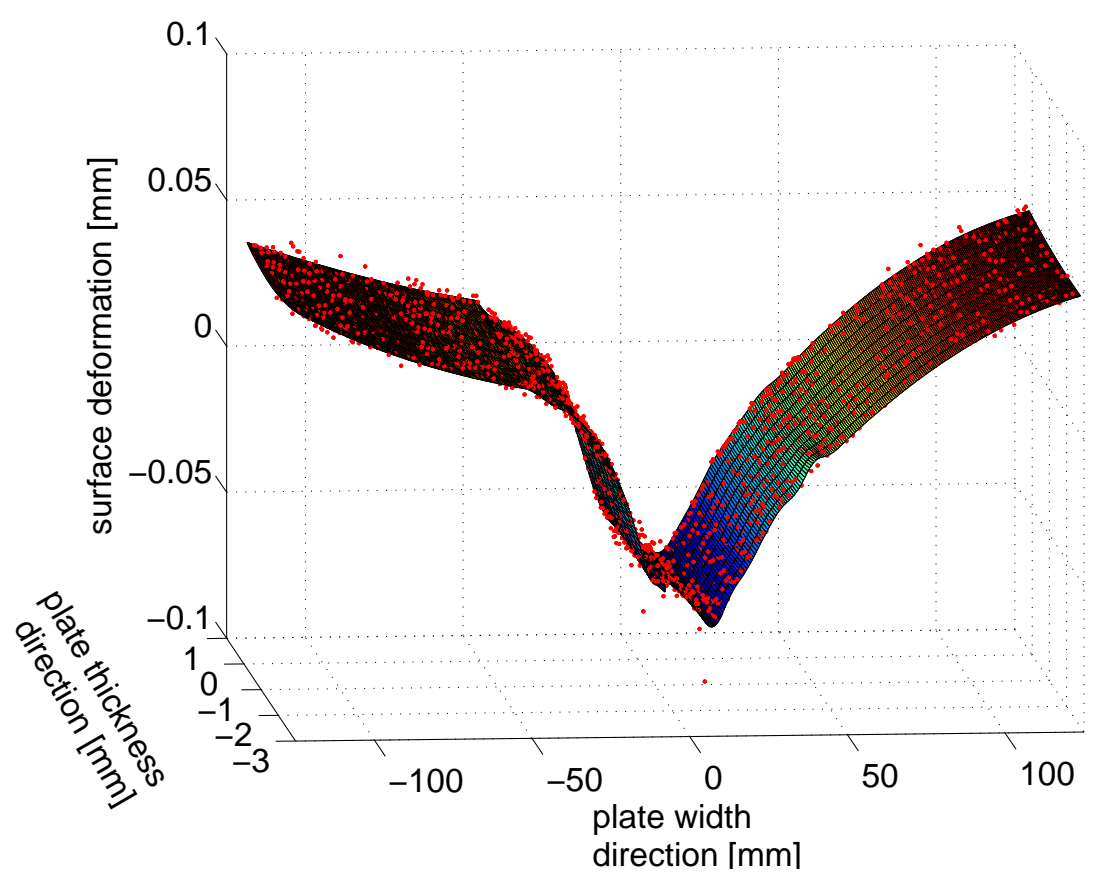

Fig. 4: Measured surface deformation after the first steps of the data conditioning process

The points for calculation of the smoothed displacement values have been defined by the node location of the finite element mesh. Table 2 shows information regarding the quality of the surface fitting procedure to the average point cloud. The goodness of fit (GOF) is calculated by equation 1 and the deviations refer to the difference on measured point coordinates of the measured and calculated smooth values. 


$$
G O F=1-\frac{\sum(\text { smooth value }- \text { original value })^{2}}{\sum\left(\text { original value }-\overline{\text { smooth value })^{2}}\right.}
$$

Table 2: Quality of the surface smoothing procedure

\begin{tabular}{cc}
\hline Parameter & Value \\
\hline Average deviation & $0.000010 \mathrm{~mm}$ \\
Maximum deviation & $0.016 \mathrm{~mm}$ \\
Standard deviation & $0.0029 \mathrm{~mm}$ \\
GOF & $99.3 \%$ \\
\hline
\end{tabular}

As can be seen, the calculated surface represents $99.3 \%$ of the average measured point cloud, and can therefore be regarded as a good fit. The calculated nodal displacements are written into an Abaqus input file for further processing

\section{FEM}

The finite element model is created in several separate steps. First the measured geometry is read into MatLab and exported in a supported format into the FEM package. Afterwards nodes and elements are created inside this package. The finalized $2 \mathrm{D}$ surface mesh is than exported into MATLAB [6] for the data smoothing procedure. The full mesh is extruded from this surface with the correct plate length and analyzed using the Abaqus solver [7].

2480 quadratic elements define the surface of the FEM model with 7957 nodes before extrusion. 60 elements are used geometrically biased by a factor of 10 in the perpendicular direction for extrusion. This leads to 649717 nodes and 148800 quadratic elements, which takes approximately two hours to be solved on a dedicated Abaqus workstation.

\section{Results}

The residual stress perpendicular to the plane of interest are retrieved from Abaqus. Figure 6(b) shows the obtained residual stress distribution on the surface of the specimen crosssection.

As can be seen, the typical M-shape usually described for the residual stress distribution in FSW is found. The maximum residual stresses are around $20 \%$ below the yield strength of the material and outside of the welding region, essentially zero and compressive residual stresses are responsible for the stress equilibrium on the surface. The plasticity induced error 
is therefore considered almost negligible.

\section{Discussion}

One doubt that may rise in the course of this work is wether the size of the modeled plate influences the results. Plate half $\mathrm{A}$ has a length of $126 \mathrm{~mm}$ and plate half $\mathrm{B}$ has a length of $180 \mathrm{~mm}$. In order to determine the influence of the difference in plate size in the finite element model, the second plate half was also modeled and the absolute difference of the obtained residual stresses is shown in Figure 5.

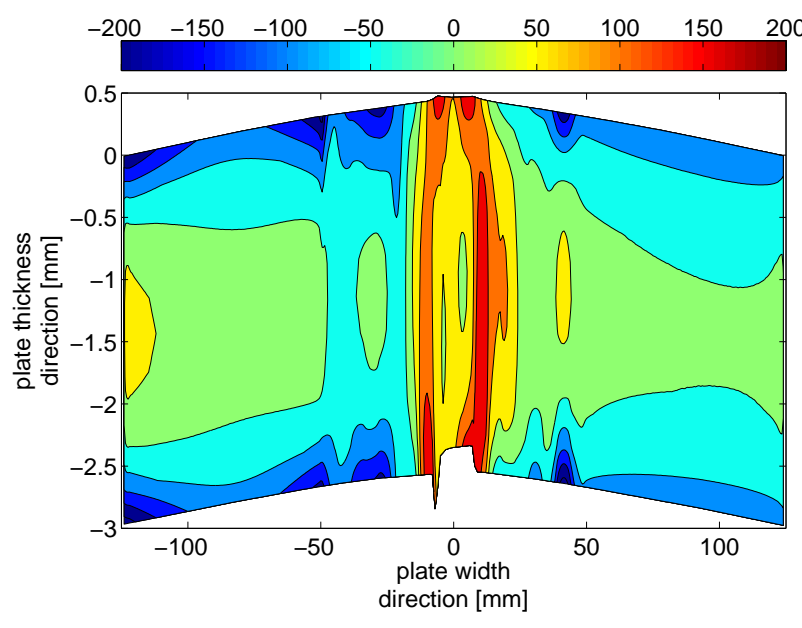

(a) plate half $\mathrm{A}$

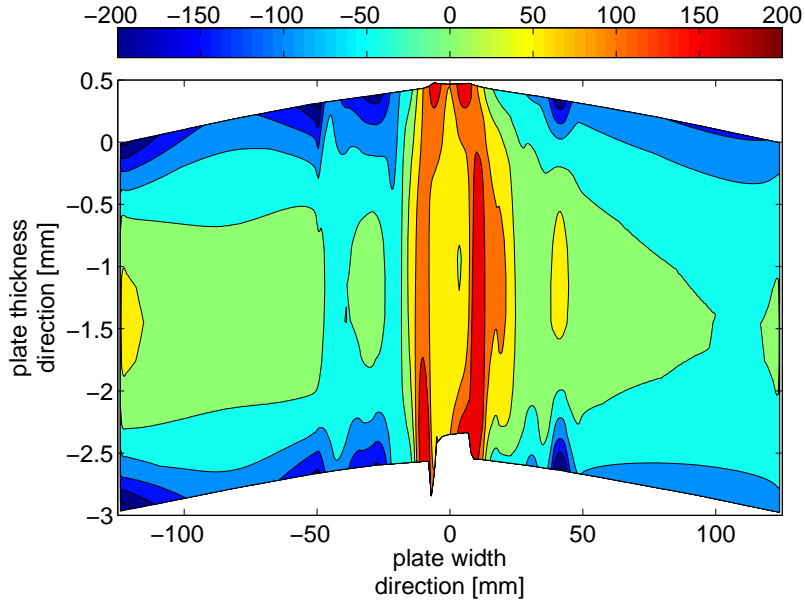

(b) plate half B

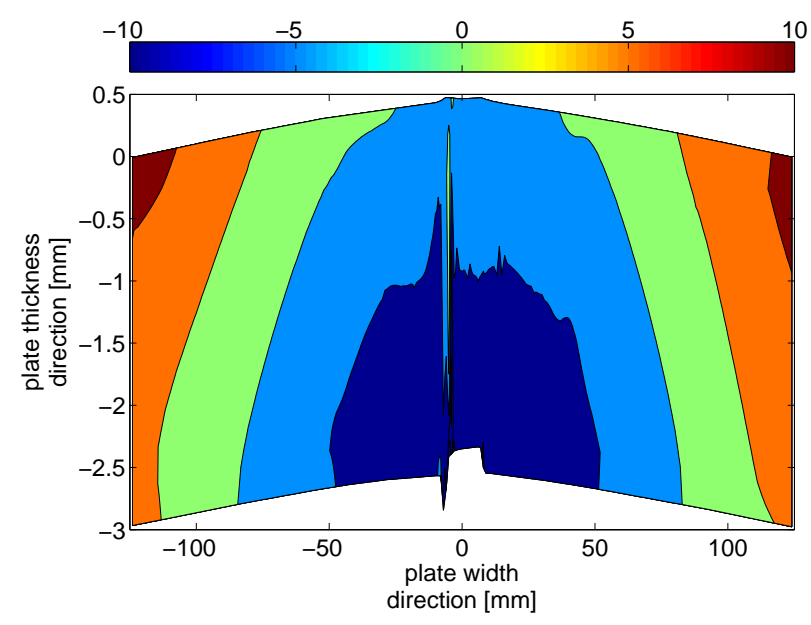

(c) absolute difference

Fig. 5: Residual stress perpendicular to the cross-section of the cutting plane calculated on both plate halves 
As can be seen, the obtained difference is relatively small, but still reaches about $10 \mathrm{MPa}$ which is equivalent to about $5 \%$ of the maximum measured stresses. In comparison to the majority of residual stress measuring techniques, this difference is almost negligible.

Penso que devemos esperar pelo input da ECRS8 antes de finalizar esta parte do texto (deadline 28 Julho); de momento o que estou a fazer é considerar a metade mais pequena, pois penso ser essa que influencia as tensões que conseguem aparecer num pedaço de material.

Measurements performed by the iHDT at 11 points on a similar specimen show comparable results to the contour method, like the M-shape, the maximum value and low compressive stresses outside of the welding nugget. Figure 6(a) shows measurements obtained using this technique compared to the results obtained using the contour method.

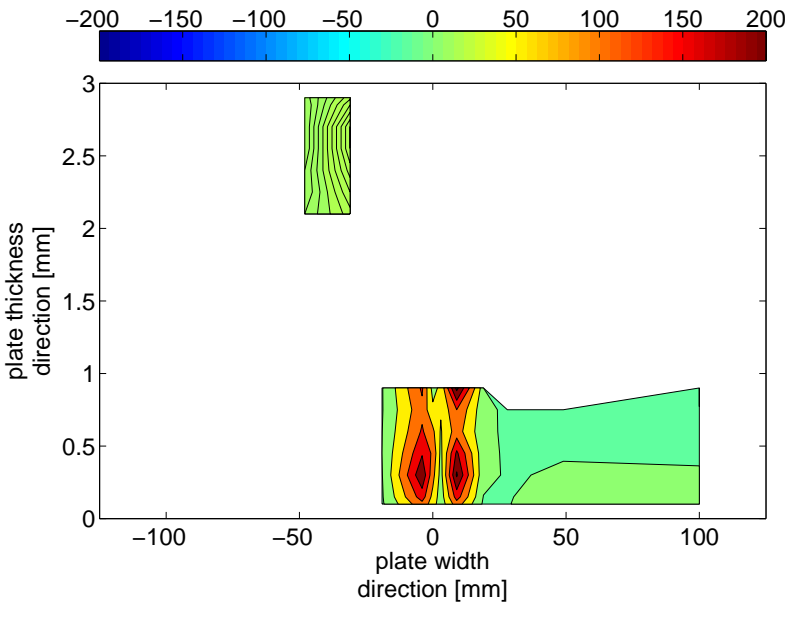

(a) iHDT

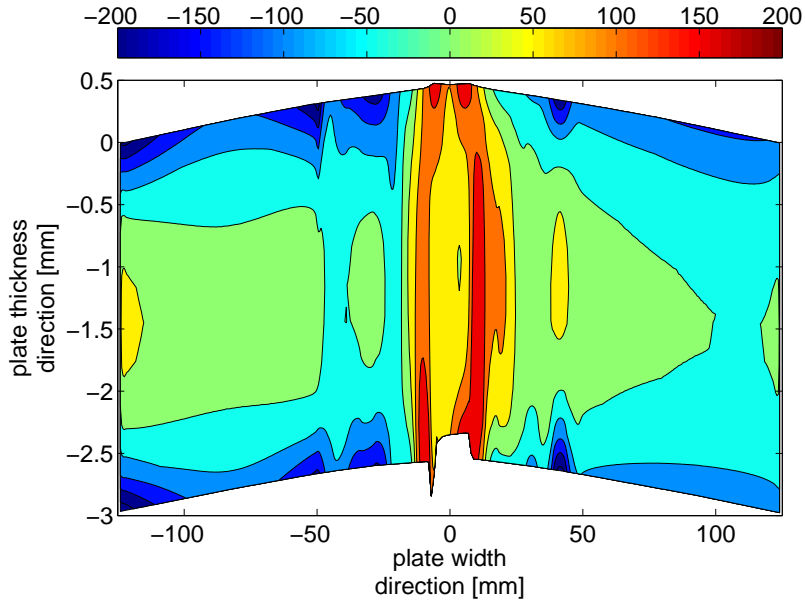

(b) contour method

Fig. 6: Residual stress perpendicular to the cross-section of the cutting plane measured by the iHDT compared to the obtained contour method results

The distribution as well as the magnitude of both measuring techniques is very similar. The highest difference is found on the borders of the surface, where the contour method predicts high compressive stresses. The strong compressive residual stresses on the borders of the cutting plane should not receive too much attention, since they are influenced by the extrapolation of the measurements performed near the border of the surface. Using a touch probe CMM such as in the present case, it is not possible to measure exactly at the border. This means that compressive residual stresses in this area may be overestimated.

The present work leads to similar results as a AA2024 FSW butt-joint measured using the synchrotron X-ray technique by Altenkirch et al. [8]. Both the magnitude and distribution are comparable, even if the cited work was performed on a $5 \mathrm{~mm}$ thick aluminium plate. For a $25 \mathrm{~mm}$ thick dissimilar FSW aluminium plate in a different alloy, a comparable stress distribution was also found by Prime et al. [9]. 


\section{Conclusions}

As could be shown, the contour method was applied successfully to a friction stir welded plate, revealing a lot of detailed information of the residual stress distribution in the inside of a friction stir weld, especially in the nugget zone.

The comparison with the proven incremental hole drilling technique shows that confidence may exist in the contour methods results. Further work in this area is therefore suggested. It was shown, that the present measurement leads to results which are similar to literature data on FSW residual stresses, although now applied to a thinner aluminium plate.

\section{Acknowledgements}

The present work was partially funded by the project PTDC/EME-TME/66362/2006 and PhD scholarship SFRH / BD / 41061 / 2007 of the Portuguese Fundação para a Ciência e Tecnologia. Dr. P. Moreira acknowledges POPH - QREN-Tipologia 4.2 - Promotion of scientific employment funded by the ESF and MCTES. The help of José Fernando Rocha Almeida is also acknowledged.

\section{References}

[1] M. Prime, Cross-sectional mapping of residual stresses by measuring the surface contour after a cut, Journal of Engineering Materials and Technology-Transactions of the ASME 123 (2001) 162-168.

[2] V. Richter-Trummer, S. M. O. Tavares, P. M. G. P. Moreira, M. A. V. de Figueiredo, P. M. S. T. de Castro, Residual stress measurement using the contour and the sectioning methods in a MIG weld: effects on the stress intensity factor, Ciência e Tecnologia dos Materiais 20 (2008) 114-119.

[3] ASTM, E837 - standard test method for determining residual stresses by the hole-drilling strain-gage method 1, ASTM Standard, 2007.

[4] S. H. Shin, Fem analysis of plasticity-induced error on measurement of welding residual stress by the contour method, Journal of Mechanical Science and Technology 19 (2005) $1885-1890$.

[5] V. Richter-Trummer, Characterization of Different Aluminium Alloys of the Series 6000 and of their Joining Processes, Mestrado integrado em engenharia mecânica, Faculdade de Engenharia da Universidade do Porto, Porto, Portugal, 2008.

[6] MathWorks, MATLAB 2009a, 2009.

[7] Abaqus version 6.7, 2007.

[8] J. Altenkirch, A. Steuwer, P. J. Withers, S. W. Williams, M. Poad, S. W. Wen, Residual stress engineering in friction stir welds by roller tensioning, Science and Technology of Welding and Joining 14 (2009) 185-192. 
[9] M. B. Prime, T. Gnaeupel-Herold, J. A. Baumann, R. J. Lederich, D. M. Bowden, R. J. Sebring, Residual stress measurements in a thick, dissimilar aluminum alloy friction stir weld, Acta Materialia 54 (2006) 4013-4021. 